\title{
A Supervoid Explanation of the Cosmic Microwave Background Cold Spot
}

\author{
F. Finelli ${ }^{1,2}$, J. García-Bellido ${ }^{3}$, A. Kovács ${ }^{4}$, F. Paci ${ }^{5}$, I. Szapudi ${ }^{6}$ \\ ${ }^{1}$ INAF-IASF Bologna, Istituto di Astrofisica Spaziale e Fisica Cosmica di Bologna \\ Istituto Nazionale di Astrofisica, via Gobetti 101, I-40129 Bologna, Italy \\ ${ }^{2}$ INFN, Sezione di Bologna, Via Irnerio 46, I-40126 Bologna, Italy \\ ${ }^{3}$ Instituto de Física Teórica IFT-UAM/CSIC, Universidad Autónoma de Madrid, Cantoblanco \\ 28049 Madrid, Spain \\ ${ }^{4}$ Institute of Physics, ELTE, 1117 Pázmány Péter sétány 1/A Budapest, Hungary \\ ${ }^{5}$ SISSA, Astrophysics Sector, Via Bonomea 265, 34136, Trieste, Italy \\ ${ }^{6}$ Institute for Astronomy, University of Hawaii, 2680 Woodlawn Drive, Honolulu, HI, 96822
}

\begin{abstract}
The Cold Spot is an anomalously cold region in the Cosmic Microwave Background (Vielva et al. 2004), either caused by a structure in the line of sight or could be of primordial origin. We search for a supervoid aligned with the Cold Spot region, filling the gap in redshift at $z<0.3$ which has never been explored in details. We find a large projected under density in the recently constructed WISE-2MASS catalogue, whose median redshift is $z \simeq 0.14$, with an angular size of 30 degrees. We show that a spherically symmetric Lemaitre-Tolman-Bondi (LTB) void model can simultaneously fit the $\delta_{\text {gal }} / b=\delta_{2 D} \simeq-0.12$ underdensity in the WISE-2MASS catalogue, and the Cold Spot as observed by both the WMAP and Planck satellites. Such an LTB supervoid gives a plausible explanation of the Cold Spot anomaly, and is preferred over the null hypothesis or a texture model.
\end{abstract}

Keywords. cosmic microwave background, observations, large-scale structure of universe

\section{Introduction}

The Cosmic Microwave Background Cold Spot (CS) (Vielva et al. 2004) could have been originated by a primordial fluctuation on the last scattering surface or by an intervening phenomenon along the line of sight. Contamination from our galaxy or by the Sunyaev-Zeldovich effect from a cluster are quite unlikely (Cruz et al. 2006), and cosmic texture hypotheses have already been tested extensively as the origin of the anomaly (Cruz et al. 2007). Alternatively, the CS could be imprinted by an intervening supervoid along the line of sight (Inoue and Silk 2007). The supervoid model has been constrained by using radio galaxies of the NVSS survey, Canada-France-Hawaii Telescope (CFHT) imaging of the CS region, redshift survey data using the VIMOS spectrograph on the VLT, and the relatively shallow 2MASS galaxy catalogue. See Finelli et al. 2014, and references therein for review. These studies are consistent with an underdensity at $z<0.3$, but they either run out of objects at low redshift, or have no redshift information to carry out a tomographic imaging of the Cold Spot area. A large area survey reaching up to $z \simeq 0.3$ is needed to map the redshift range unconstrained by previous studies. For this purpose, we use the recently produced WISE-2MASS galaxy catalog (Kovács and Szapudi 2014), with median redshift of $z \simeq 0.14$, and sky coverage of 21,200 square degrees after masking dusty regions. Galaxy samples, however, are biased tracers of the underlying dark matter distribution. We thus measured and modeled the angular power spectrum of WISE-2MASS galaxies, and found a galaxy bias $b_{g}=1.41 \pm 0.07$, that we take into account in our further analyses. We show the projected galaxy density profile 
around the CS in the left panel of Fig. 1. Measurement errors are due to Poisson fluctuations calculated from the expected number of galaxies in a ring or a disk. We detect an underdensity with high significance. At larger radii, the radial profile is consistent with a supervoid surrounded by a gentle compensation that converges to the average galaxy density at $\sim 50^{\circ}$. The supervoid might also contain a deeper inner void, with its own compensation at around $\simeq 8^{\circ}$. See Finelli et al. 2014 for details.

\section{Modeling the supervoid}

Next we build a $\Lambda$ LTB void model for the underdensity (Garcia-Bellido and Haugbølle 2008), characterized by a spatial curvature profile $k(r)=k_{0} r^{2} \exp \left(-r^{2} / r_{0}^{2}\right)$, written as a linear metric perturbation in $\Lambda \mathrm{CDM}$,

$$
\Phi(\tilde{r})=\Phi_{0} \tau^{2} e^{-\frac{\tilde{r}^{2}}{\tilde{r}_{0}^{2}}},
$$

with the LTB radius $r$ related to the co-moving FRW radius through $\tilde{r}=\sqrt{3 / 4 \pi} H_{0} r$, and $\tau$ conformal time. This scalar potential gives rise to a $3 \mathrm{D}$ density profile for the void

$$
\delta_{3 D}(\tilde{r})=-\delta_{0}\left(1-\frac{2 \tilde{r}^{2}}{3 \tilde{r}_{0}^{2}}\right) e^{-\frac{\tilde{r}^{2}}{\tilde{r}_{0}^{2}}},
$$

characterized by two parameters, the co-moving width $\tilde{r}_{0}$ and the depth $\delta_{0}$. The 3D density (2.2) is then projected onto the transverse plane, using the WISE window function

$$
\delta_{2 D}(\theta)=\int_{0}^{\infty} \delta_{3 D}(\tilde{r}(y, \theta)) y^{2} \phi(y) d y .
$$

We also compute the linear Integrated Sachs-Wolfe and the non-linear Rees-Sciama effects on the CMB. For a large compensated void with a profile of Eq. 2.2, the linear ISW effect is dominated by the non-linear Rees-Sciama effect,

$$
\delta T(\theta)=-A\left(1-\frac{28}{13} \frac{\theta^{2}}{\tilde{\theta}_{0}^{2}}\right) e^{-2 \frac{\theta^{2}}{\tilde{\theta}_{0}^{2}}},
$$

where $\tilde{\theta}_{0}=\sqrt{3 / 4 \pi} \theta_{0}$, and $\tan \theta \simeq \theta$, is applied. The magnitude of the decrement depends on the parameters of the void,

$$
A=51.0 \mu \mathrm{K}\left(\frac{r_{0}}{155.3 h^{-1} \mathrm{Mpc}}\right)^{3}\left(\frac{\delta_{0}}{0.2}\right)^{2},
$$

and $\theta_{0}=\left(180^{\circ} / \pi\right)\left(r_{0} / d_{A}\left(z_{0}\right)\right)$, with $d_{A}(z)$ the angular diameter distance in a flat $\Lambda \mathrm{CDM}$ model $\left(\Omega_{\mathrm{M}}=0.3, h=0.7\right)$, and $z_{0}$ the redshift of the center of the void, at co-moving distance $y_{0}=y\left(z_{0}\right)$. Note that the LTB model is only used as a general relativistic model for the void dynamics and its effect on the CMB, while the background cosmology is assumed to be standard $\Lambda$ CDM.

A large LTB supervoid is sufficient to explain a colder spot in the CMB of about half the size, or in other words a shallower supervoid is sufficient to imprint the same temperature depression in the $\mathrm{CMB}$, than is allowed for a supervoid assuming $\Lambda \mathrm{CDM}$ cosmology. Note that the LTB model is only used as a general relativistic model for the void dynamics and its effect on the $\mathrm{CMB}$, while the background cosmology is assumed to be standard $\Lambda$ CDM. For detailed measurements, a $\chi^{2}$ statistic is constructed. We perform a simultaneous fit for the projected LTB void in the WISE-2MASS map, and the corresponding temperature depression effect in the CMB data, with parameters $\delta_{0}, r_{0}$, 

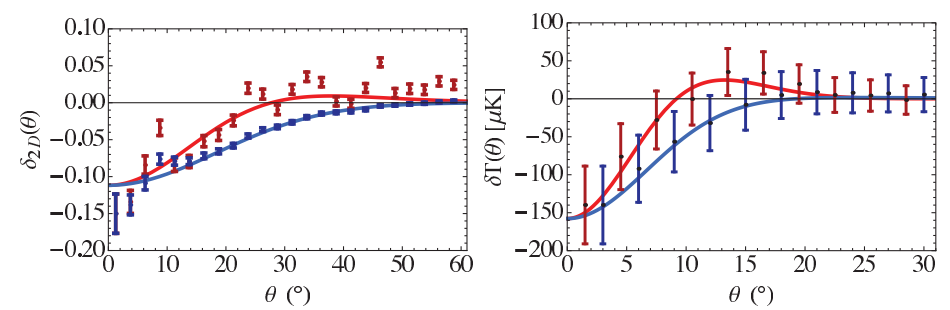

Figure 1. The density profile from WISE-2MASS catalogue compared with the theoretical model for the underdensity (2.3) (left panel). The temperature profile from Planck SMICA map (right panel) (Ade et al. 2013) compared with the predicted signal (2.4). The red (blue) lines are the theoretical profiles for rings (disks) and in dark red (blue) are the measurements.

and $z_{0}$. The first term corresponds to the $\chi^{2}$ of the projected LTB void profile (2.3) using uncorrelated Poisson errors, $\sigma_{i}$. The second term is the $\chi^{2}$ of the CMB profile compared with the LTB prediction (2.4) of the void observed in WISE-2MASS. The best fit LTB void parameters we have found are $\delta_{0}=0.25 \pm 0.10(1 \sigma), r_{0}=(195 \pm 35) \mathrm{h}^{-1} \mathrm{Mpc}(1 \sigma)$, and $z_{0}=0.16 \pm 0.04(1 \sigma)$. The LTB model parameter $\delta_{0}$ is the 3D dark matter density, giving a $12 \%$ projected underdensity at the center of the void, i.e. $\delta_{2 \mathrm{D}}(\theta=0)=-0.12$. The angular sizes $\theta_{0}=28.8^{\circ} \pm 5.2^{\circ}$, and $\tilde{\theta}_{0}=14.1^{\circ} \pm 2.5^{\circ}$ are derived parameters, which correspond to angular scales of the profile on the galaxy map and the CMB, respectively. For later comparison, we calculate the averaged underdensity within the best fit radius $r_{0}=195 h^{-1} \mathrm{Mpc}$. The 3D top-hat-averaged density from the LTB profile, see Eq. (2.2), is $\bar{\delta}=3 / r_{0}^{3} \int_{0}^{r_{0}} r^{2} d r \delta(r)=-\delta_{0} / e$. This finally gives the average void depth $\bar{\delta}=-0.10 \pm 0.03$.

\section{Conclusions}

We have found a super void aligned with the CS in the WISE-2MASS catalogue and shown for the first time that both the supervoid profile and the CMB profile can be simultaneously fit assuming an LTB profile embedded in an FRW universe located at redshift $z=0.155 \pm 0.037$ with radius $r_{0}=(195 \pm 35) h^{-1} \mathrm{Mpc}$, and top-hat-averaged depth $\bar{\delta}=-0.10 \pm 0.03$. Such an LTB supervoid gives a perfect explanation, via a ReesSciama effect, of the Cold Spot anomaly, and is strongly preferred (using a Bayesian analysis) over the null hypothesis (statistical fluctuation) or a texture model. Note that these values are in excellent agreement with the findings of Szapudi et al. 2014, who combined the WISE-2MASS galaxy data with Pan-STARRS1, optical observations for a direct tomographic imaging of the CS region.

\section{References}

Vielva P., Martinez-Gonzalez E., Barreiro R. B., Sanz J. L., \& Cayon L., 2004, Astrophys. J. 60922

Cruz M., Tucci M., Martinez-Gonzalez E., \& Vielva P., 2006, Mon. Not. Roy. Astron. Soc. 369 57

Cruz M., Turok N., Vielva P., Martinez-Gonzalez E.,, \& Hobson M., 2008, Mon. Not. Roy. Astron. Soc. 390 (2008) 913

Inoue, K. T. \& Silk, J. 2006, ApJ, 648, 23-30

Szapudi, I., Kovács, A., Granett, B. R., et al. 2014, arXiv:1405.1566

Finelli, F., Garcia-Bellido, J., Kovacs, A., Paci, F., \& Szapudi, I. 2014, arXiv:1405.1555

Kovács, A. \& Szapudi, I. 2014, arXiv:1401.0156

Garcia-Bellido, J. \& Haugbølle, T. 2008, JCAP, 4:3

Ade P. A. R., et al. [Planck Collaboration], 2013, arXiv:1303.5072 [astro-ph.CO]. 